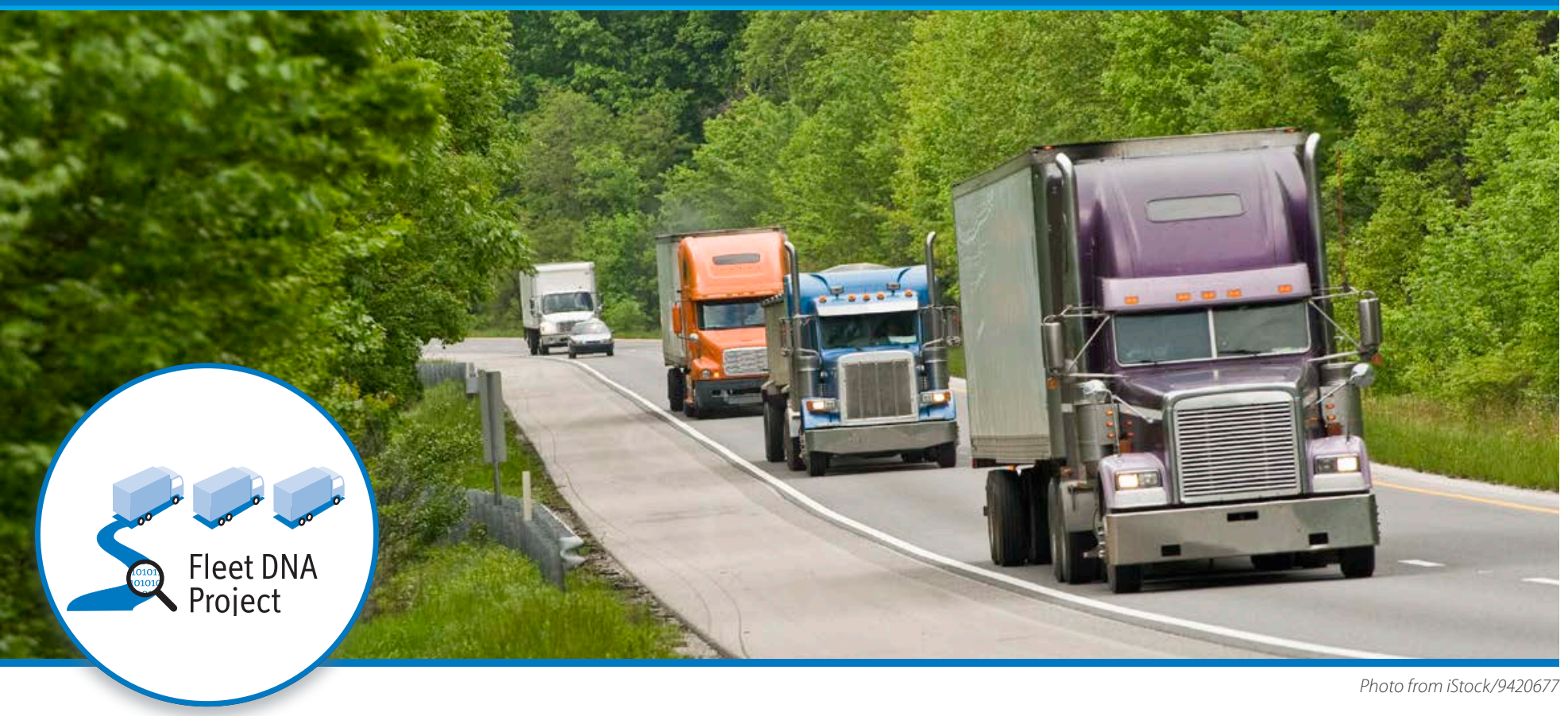

The Fleet DNA Project aims to accelerate the evolution of advanced vehicle development and support the strategic deployment of market-ready technologies that reduce costs, fuel consumption, and emissions. At the heart of the Fleet DNA Project is a clearinghouse of medium- and heavy-duty commercial fleet transportation data for optimizing the design of advanced vehicle technologies or for selecting a given technology to invest in. Designed by the U.S. Department of Energy's National Renewable Energy Laboratory in partnership with Oak Ridge National Laboratory, this online tool will help vehicle manufacturers and fleets understand the broad operational range for many of today's commercial vehicle vocations.

\section{Transportation Data}

Now in the early stages of development, this benchmarking tool will offer access to easy-to-interpret data summaries and graphical data outputs based on real-world "genetics" from medium- and heavy-duty commercial fleet vehicles operating within a variety of vocations. These summaries will be based on thousands of data points of operation per vocation, with an initial focus on transit buses, school buses, delivery vehicles (Class 3 and 5), regional tractors, utility trucks, cabs, and sleeper-cab trucks.

The Fleet DNA Project will feature transportation data that a variety of partners gathered from various fleet evaluation and research projects, particularly those that focus on the vocations and vehicles that consume the most fuel in the United States.

A wide range of statistics, compiled for each vocation and/or vehicle type, will be publically available. More detailed data and specialized analysis could be accessible to partners who contribute data or provide additional support. Example data sets might contain information such as aggregated route distance, average speed, maximum acceleration, stops per mile, load and grade statistics, and many other drive cycle metrics along with comparisons to standard industry test cycles. Users will be able to produce customized datasets using the Fleet DNA tool. 


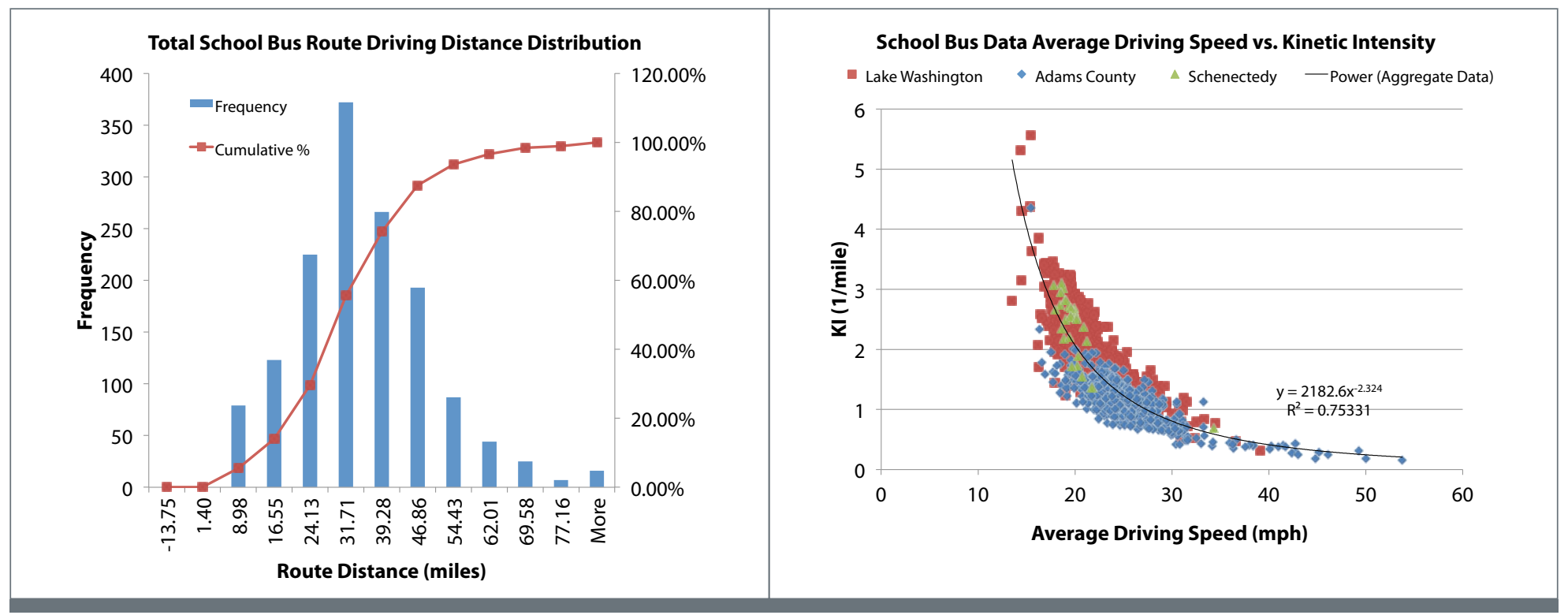

These two graphs serve as examples of the type of vocational data summaries that will be available in the Fleet DNA clearinghouse. The graph on the left provides information about daily route schedules for school buses and the graph on the right highlights driving speed versus kinetic intensity.

\section{Data Safeguards}

Building on the success of the light-duty-focused Transportation Secure Data Center, the Fleet DNA Project will feature similar safeguards to protect proprietary data while providing access to cleansed, filtered, and analyzed data summaries. Detailed data on specific fleets will not be revealed; data will remain anonymous, focusing only on vehicle and vocation type and/or location.

\section{Interested in Participating?}

NREL works with a variety of organizations that use and/or contribute data. Contact Kevin Walkowicz at 303.275.4492 or Kevin.Walkowicz@nrel.gov for more information about the project or to discuss partnership opportunities.

\section{About NREL's Transportation R\&D}

Dedicated to renewable energy and energy efficiency, NREL is at the forefront of R\&D for tomorrow's sustainable transportation solutions. NREL's innovative approach helps government, industry, and other research partners develop high-performance, low-emission, fuel-efficient vehicles, components, and systems. 